\title{
INTER, MULTI, Y TRANSDISCIPLINARIEDAD DEL TURISMO
}

\author{
Isabel Marcano Anés* \\ https://orcid.org/0000-0002-4677-4656
}

RECIBIDO: Julio 2020 / ACEPTADO: Agosto 2020 / PUBLICADO: Septiembre 2020

Como citar: Marcano Anés, Isabel. (2020). Inter, multi, y transdisciplinariedad del turismo. Telos: revista de Estudios Interdisciplinarios en Ciencias Sociales, 22 (3), Venezuela. (Pp.614-625).

DOI: www.doi.org/10.36390/telos223.10

\section{RESUMEN}

A lo largo del tiempo, el estudio del turismo ha comenzado a partir de diferentes disciplinas sociales como la economía, sociología, psicología, antropología, geografía, entre otras, por lo que se han utilizado abordajes inter, multi y transdisciplinarios con la finalidad de conocer y comprender lo que es realmente el turismo. Cabe destacar que, existen diversas definiciones sobre turismo las cuales varían entre sí, dependiendo del punto de vista que se le da al concepto (ya sea como fenómeno, actividad, ciencia, entre otros) o el ámbito de formación de quien lo define (economía, sociología, psicología, estadística, geografía, entre otras), situación que ha llevado a plantear diversos abordajes para estudiar el turismo. Con este artículo se busca describir la inter, multi y transdisciplinariedad del turismo, a fin de determinar cuál de esos abordajes es el más adecuado para lograr entender la naturaleza del conocimiento turístico. Entre los autores consultados para el desarrollo de este artículo están Moreira, López y De Abreu (2015), Rocha Centeno (1992), Castillo y Lozano (2006), Castaño (2005), Campodónico y Chalar $(2010,2017)$, entre otros. Para ello se consideró el diseño de investigación documental, basado en la hermenéutica analítica la cual permite interpretar la visión de los distintos autores y/o estudiosos que han abordado el tema y poder reconocer la perspectiva más idónea para conocer la naturaleza del conocimiento turístico. Se logró determinar que con el abordaje interdisciplinario se puede acercar a un poco a conocer la naturaleza del conocimiento turístico, sin embargo, el más adecuado es el transdisciplinario ya que este aborda, de una forma más integral, los diversos aspectos y enfoques que abarcan el conocimiento turístico.

Palabras Claves: disciplinas, multidisciplinariedad, interdisciplinariedad, transdisciplinariedad, conocimiento turístico.

\footnotetext{
* Departamento de Turismo, Universidad de Oriente, Núcleo Nueva Esparta, Guatamare, Venezuela. Correo electrónico: icma16@gmail.com
} 


\title{
Inter, multi, and transdisciplinarity of tourism
}

\begin{abstract}
Over time, the study of tourism has started from different social disciplines such as economics, sociology, psychology, anthropology, geography, among others, which is why inter, multi and transdisciplinary approaches have been used in order to know and understand what is the tourism. Notably, there are various definitions of tourism which vary among themselves, depending on the point of view that is given to the concept (whether as a phenomenon, activity, science, among others) or the scope of training of the person who defines it (economy, sociology, psychology, statistics, geography, among others), this situation has led to proposing various approaches to study tourism. This article seeks to describe the inter, multi and transdisciplinary of tourism, to determine that approach is the most appropriate to understand the essence of tourism knowledge. Among the authors consulted for the development of this article are Moreira, López and De Abreu (2015), Dos Santos (2009), Rocha Centeno (1992), Castillo and Lozano (2006), Castaño (2005), Campodónico and Chalar (2010, 2017), Morin (2001), among others. For this purpose, the design of documentary research was considered, based on the analytical hermeneutics, which allows to interpret the vision of different authors and / or scholars who have approached the subject and to be able to recognize the most suitable perspective to understand the essence of the tourist knowledge. It was determined that with the interdisciplinary approach it is possible to get a little closer to knowing the essence of tourist knowledge, however, the most appropriate is the transdisciplinary one since it addresses, in a more comprehensive way, the various aspects and approaches that cover the tourism knowledge.
\end{abstract}

Keywords: disciplines, multidisciplinarity, interdisciplinarity, transdisciplinarity, tourist knowledge.

\section{Introducción}

Moreira, López, y De Abreu (2015, p. 451), señalan que "aunque la ciencia del turismo esté cargada aun de preconceptos por parte de intelectuales de diversas áreas del conocimiento que acreditan que no es digna de estudios e investigaciones, el turismo se afirma como tal". Por lo tanto, esto debe ser un desafío para los especialistas de las distintas áreas del conocimiento que están orientados a analizar la finalidad del saber turístico, es decir, las relaciones, interacciones y procesos que se originan por el ser humano al desplazarse. Múltiples disciplinas han incidido e inciden en el estudio del turismo, el cual es un fenómeno complejo producto de múltiples interrelaciones, actores, procesos y contextos, que han dificultado comprender su base conceptual.

La relevancia que tiene el turismo en el ámbito de la economía mundial ha hecho que muchos investigadores desde distintas disciplinas se acerquen al turismo para estudiarlo como un fenómeno multidisciplinar que ha generado y genera discusiones desde el punto de vista científico (Acosta y Garcia, 2016). Hay que resaltar que, desde hace años se viene discutiendo sobre la definición del turismo y su carácter científico, como fenómeno independiente Ash y 
Turner consideran que el turismo no tiene contenido científico (tendencia a científica); mientras que Hunzinker afirma que el turismo es una ciencia (tendencia científica); y, Sessa y Boullón resaltan la tendencia multidisciplinaria que caracteriza al turismo (Martínez, 2013), todo esta situación genera muchas controversias a la hora de interpretar el conocimiento turístico.

Se puede inferir que el turismo cuenta con los elementos necesarios que le dan carácter científico, sin embargo, es necesario dejar de lado la rigurosidad que caracteriza la visión positivista que ha prevalecido en las distintas investigaciones que se han realizado, y abrir camino a la visión interpretativa. La necesidad de construir un conocimiento científico sobre el turismo requiere nuevos puntos de vista en los complejos análisis de su objeto de estudio, desde una visión más integradora y comprometida con una área del saber autónoma desde su concepción, independiente desde sus aportes, científica desde su forma de analizar la realidad y compleja desde sus participantes (Acosta y García, 2016).

Desde abordajes inter, multi y transdisciplinarios se ha intentado explicar realmente lo que es el turismo. Cabe destacar que, los primeros estudios referentes al turismo estaban enfocados en la economía, interpretándolo desde la ley de oferta - demanda, la producción y el consumismo; luego se basó en la sociología, destacando las motivaciones y la conducta de los turistas, así como en los aspectos positivos y negativos que genera; también se realizaron estudios relacionados con la estadística (afluencia de turista, ingresos, entre otros), la antropología, la psicología, la geografía e incluso la política, sin embargo, dichos estudios han estado basados bajo los parámetros propios de cada disciplina, dificultando así el entendimiento integral del conocimiento turístico.

Es evidente el conjunto de disciplinas que hacen vida en el turismo, por lo que es necesario un adecuado abordaje que permita conocer y comprender la definición de este fenómeno socioeconómico. Considerando lo anterior, este artículo está centrado en analizar cómo ha sido abordado el estudio del turismo, tomando en cuenta específicamente la inter, multi y transdisciplinariedad del mismo.

Se desarrolló una investigación de tipo documental, lo que permitió comprender los abordajes disciplinarios que han incidido e inciden en el estudio del turismo. En este sentido, a través de la aplicación de un diseño documental, con un enfoque hermenéutico analítico, se interpretan diferentes autores y/o estudiosos que han planteado sus perspectivas en relación a la interpretación del turismo en función de las distintas disciplinas. Primero, se presenta la interdisciplinariedad del turismo, destacando las apreciaciones de Morales (2011), Campodónico y Chalar (2017), Goelner, Ritchie y Mclnstosh (2002) y Rocha Centeno (1992); luego se expone el abordaje multidisciplinario del mismo, con los aportes de Santos Filho (2009), Rocha Centeno (1992), Castillo y Lozano (2006), Castaño (2005), Martini (2013), Moreira, López y De Abreu (2015), Campodónico y Chalar (2017);y por último, su transdisciplinariedad, con los planteamientos de Campodónico y Chalar (2010, 2017), Rocha Centeno (1992), La Rosa (2012), Morin (2001) y Panosso (2007).

\section{Interdisciplinariedad del Turismo}

Desde siempre el turismo ha sido una materia de estudio que requiere aproximaciones a partir de diversas disciplinas, sin embargo, a pesar de ser analizado desde múltiples campos, la mayoría de las investigaciones centralizan sus temas en áreas particulares, lo que trae como consecuencia que los resultados se parcialicen, dejando de lado el enfoque holístico del fenómeno y demostrando la carencia de interdisciplinariedad (Morales, 2011). 
Para concebir al Turismo como área de estudio es imperante considerar una postura interdisciplinaria. Hay que destacar que, la necesidad de que la investigación en turismo sea abordada de forma interdisciplinaria ha sido reconocida por múltiples autores (Ascanio 2010, Castillo 2005, De La Cruz et al 2008, Barreto y Otamendi 2010).

La interdisciplina se considera un espacio para distintos modos de conocimiento que posibilitan la interacción de esas disciplinas, de forma tal que alcance una mayor amplitud, partiendo de lo múltiple, complejo, diverso, entre otras características (Campodónico y Chalar, 2017).

El turismo se caracteriza por ser complejo, comprender un conjunto de procesos e interacciones, diversas disciplinas, así como la participación de múltiples actores, convirtiéndolo en un fenómeno que ha sido difícil de entender. Los diversos abordajes que han sido estudiados deben considerarse de forma entrelazada, donde cada disciplina aporte los elementos necesarios para estudiarlo de forma integral y complementaria.

Goeldner, Ritchie y McIntosh (2002), en cuanto al abordaje interdisciplinario enfatizan que debido a lo amplio, complejo y multifacético que es el turismo, es indispensable distintos puntos de vista para estudiar ese campo, claro está, cada uno adecuado a un propósito u objetivo diferente.

Existen diferentes disciplinas relacionadas con el estudio del turismo, por lo tanto, es necesario definir las áreas comunes de éstas con el turismo, y determinar las relaciones existentes, las teorías y métodos que utilizan y que pueden ser consideradas dentro del estudio y análisis del turismo, lo que hace evidente que este fenómeno tan complejo y multifacético, asuma un papel interdisciplinar en el mundo académico (ver figura 2).

Figura 1. Interdisciplinariedad del Turismo.

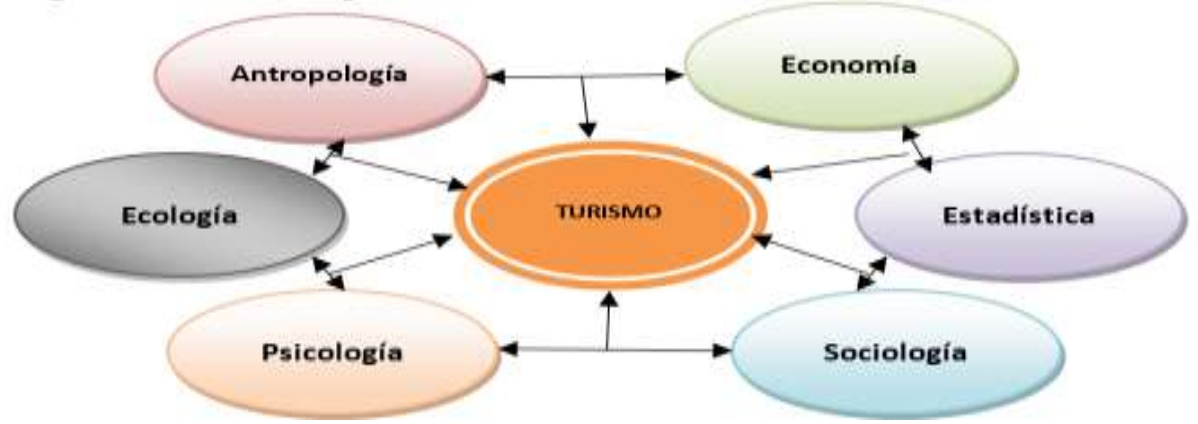

Fuente: Elaboración propia.

El turismo requiere para su estudio de un acercamiento integral e interdisciplinario que tome en cuenta las influencias externas e internas que intervienen en su desarrollo, así como todos los procesos multidinámicos que éste origina. Por lo que, puede apoyarse en los métodos y teorías de las distintas disciplinas que abarcan la realidad turística, y así puedan surgir, en función de la interacción de las disciplinas, algunas bases conceptuales, teóricas y metodológicas propias del turismo; por lo tanto, desde un abordaje interdisciplinario se podrá 
acercar a este objetivo y a su vez permitirá una aproximación para comprender la base del conocimiento turístico.

\section{Multidisciplinariedad del Turismo}

Para Dos Santos (2009, p. 6), el turismo "busca la multidisciplinariedad en el estudio de su objeto y trabaja en la construcción de una explicación capaz de resistir procedimientos de prueba reconocidos...; Ese objeto constituye una formación económico-social determinada, específica y particular, que posee determinaciones propias...".

Como se puede apreciar, a través de la multidisciplinariedad se busca explicar el objeto del turismo el cual logra ser visto como fenómeno social dentro de las especificaciones económicas, políticas, sociales y culturales, pero constituidas en una formación determinada, específica y particular, que logra ser abordado desde una parcialidad de la realidad (ver figura 1).

Figura 2. Multidisciplinariedad del Turismo.

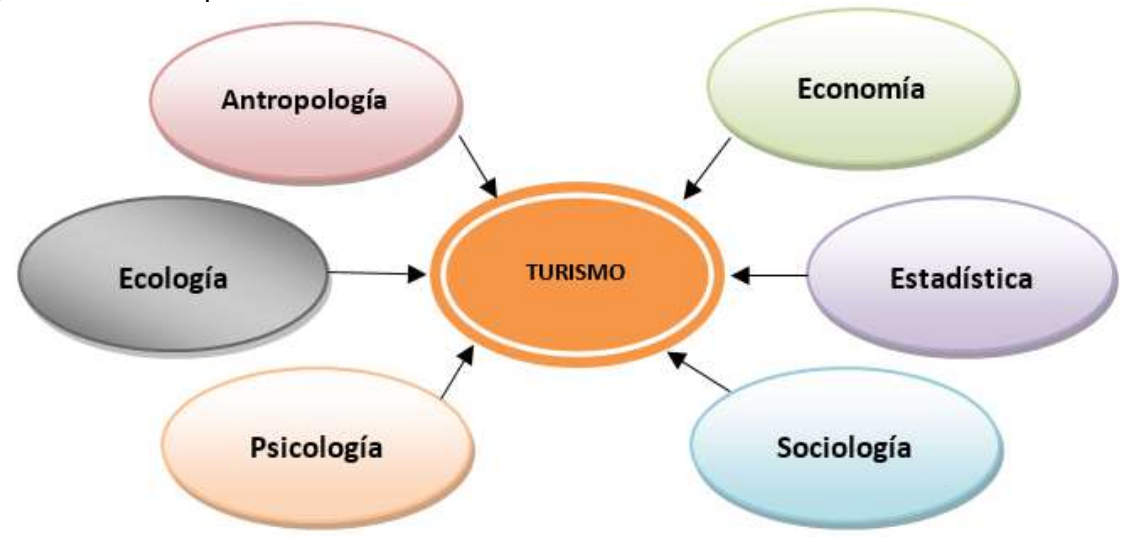

Fuente: Elaboración propia.

El estudio del turismo se ha caracterizado por la confluencia de diversas disciplinas científicas (economía, sociología, psicología, geografía, entre otras) que han tratado de definirlo, describirlo, explicarlo, interpretarlo, comprenderlo y predecirlo, para ello lo han abordado considerando sus propias técnicas, metodologías y procedimientos. "El turismo es multidisciplinario. Lo multidisciplinario es una yuxtaposición de diversas disciplinas que se estudian sin establecer relaciones entre ellas" (Rocha Centeno, 1992, p. 58). Por lo tanto, en el turismo diferentes disciplinas - con diversas perspectivas -han estudiado desde la visión particular de cada una, lo cual comprende la división de territorios entre los saberes, representando esto un gran inconveniente, debido a que su análisis se ha fragmentado e interpretado tomando en cuenta los intereses particulares de esas disciplinas.

Por ejemplo, el estudio del turismo es visto como un negocio desde la economía, en la geografía en función de la relación del hombre con su espacio, en la psicología por la conducta del individuo, en la antropología por las relaciones culturales del hombre y en la historia por los hechos y las manifestaciones (Castillo y Lozano, 2006). 
En este mismo orden de ideas, Rocha Centeno (1992), expone que entre las disciplinas que estudian el turismo se encuentran:

- Sociología, a través de ella es posible logra el acercamiento a las causas sociales del fenómeno turístico.

- Economía, por la generación de gastos originados por el desplazamiento del turista y que son captados como ingresos que al ser administrados pueden generar empleos e inversiones.

- Psicología, permite conocer las razones y/o motivos por los cuales viaja el turista.

- Antropología, que plantea las teorías que permiten conocer la naturaleza que impulsa al turista a viajar y como esa decisión de viaje altera la estructura económica, social y cultural tanto del lugar de origen como de destino.

- Ecología, disciplina que aborda la relación de los flujos turísticos con la degradación del ambiente en los lugares turísticos muy visitados.

- Matemáticas, que permiten realizar los análisis cuantitativos del fenómeno turístico.

- Tecnología, instrumento importante en el fenómeno turístico, por ejemplos los sistemas computarizados de reservación en las distintas empresas turísticas.

Es evidente, como las disciplinas han abordado el estudio del turismo, desde sus características propias sin establecer relaciones con otras disciplinas, demostrando esto la parcialidad en su interpretación. Cabe destacar también que, incluso algunas disciplinas se han especializado particularmente en el área de turismo. En relación a esto, Castaño (2005), plantea:

- La psicología del turismo, considerada como una disciplina científica de carácter más bien aplicado, cuyo objeto de estudio lo constituyen el comportamiento y las interacciones de los individuos en relación con los procesos mentales y afectivos, así como la influencia que ejercen los procesos sociales de los contextos turísticos donde dichas conductas se manifiestan.

- La antropología del turismo abarca tres ámbitos de estudio: a) el individuo fuera de su entorno habitual, b) la industria que responde a sus necesidades y, c) el impacto, tanto él como la industria, ocasionan sobre el ámbito físico, económico y sociocultural de las poblaciones anfitrionas.

- La geografía del turismo gira principalmente en torno a tres aspectos: a) los problemas espaciales generados por el turismo, b) la comparación de regiones turísticas con perfiles similares y estadísticas diferentes, y c) la planificación y creación de un destino turístico.

Estos tratamientos disciplinarios del turismo impiden ver lo global del turismo, es decir, el sistema turístico (que ella parcializa) así como el sujeto turístico (que ella disuelve) y la extensa subdivisión en disciplinas que hace difícil reflexionar sobre lo complejo (Martini, 2013), que caracteriza al turismo, por lo tanto, el abordaje multidisciplinario no es adecuado a la hora de conocer la naturaleza del conocimiento turístico.

Como se puede apreciar, en el estudio del turismo la corriente tradicional ha originado conocimiento producto de una multidisciplinariedad acrítica y descriptiva donde las categorías y conceptos no están entrelazados en modelos adecuados para el fenómeno turístico, esto trae como consecuencia que no se planteen opciones para elaborar y ejecutar metodologías de investigación propias y específicas de las realidades objeto de estudio, además de las imprecisiones terminológicas (Castillo y Lozano, 2006). Los estudios turísticos que se han abordado desde la perspectiva de cada disciplina, se han realizado bajo las condiciones, 
definiciones y metodologías propias de la realidad de la disciplina como tal, y no relacionados con el fenómeno turístico, lo que representa una debilidad.

Lo anterior, ha generado un desequilibrio de lo turístico entre sus hechos y el estatus de su conocimiento, por lo que, cuando se ha investigado en lo turístico únicamente se limita a la descripción de sus manifestaciones dejando de lado el análisis de su naturaleza, el fundamento de sus conocimientos sobre el cual se construye y lo que se concibe como teoría (Castillo y Lozano, 2006). No podemos limitarnos a estudiar el turismo, desde las visiones particulares de cada disciplina (desde la multidisciplinariedad), se debe ir más allá para poder comprender la esencia de este fenómeno.

Es evidente que, el turismo está en constante proceso de construcción social y admite características específicas acorde al contexto socio - histórico donde se desarrolla, por lo tanto, los problemas complejos son inherentes al turismo. Por tal motivo, no puede reducirse el objeto de estudio del turismo no es posible reducirlo "a la simple yuxtaposición de situaciones o fenómenos que pertenezcan al dominio exclusivo de una disciplina" (García citado por Campodónico y Chalar, 2017, p. 462), sino que se debe ir más allá, buscar las relaciones que permitan comprender al fenómeno turismo, tomar en cuenta la diversidad de disciplinas que hacen vida en el turismo, pero no limitarse a la particularidad de cada una de ellas sino ir más allá, establecer las distintas relaciones que se originan entre las mismas para poder comprender lo que es realmente el turismo.

Desde este abordaje, la multidisciplinariedad, el turismo carece de cuerpo teórico y metodología de investigación propia. El cuerpo teórico está vinculado con disciplinas diversas y en muchos casos surgen ciertas confusiones entre las mismas al abordar un mismo aspecto de la realidad turística, y la metodología de investigación desarrollada siempre está enfocada en la visión particular de cada disciplina y ésta se aleja, en la mayoría de los casos, de la peculiaridad que caracteriza al turismo. Por lo anterior, este abordaje no se considera el más adecuado para comprender el conocimiento turístico, ya que tiene como base las visiones particulares de cada disciplina.

Hay que resaltar que, para comprender la naturaleza del conocimiento turístico debe existir correlación entre el sujeto y el objeto de conocimiento; y para que esta correlación se desarrolle son necesario el sujeto cognoscente, el objeto de conocimiento y la imagen. Es imperante que, el sujeto cognoscente salga de su esfera psicológica e ingrese a la esfera ontológica del objeto de conocimiento y capture sus elementos para retornar a ésta y originar una imagen que refleje los elementos mientras ésta permanece significativa al sujeto (Hessen, 2001). Por lo tanto, si el proceso de conocimiento se desarrolla desde la visión particular de cada disciplina el resultado será un conocimiento parcializado del turismo, por lo que el abordaje multidisciplinario no es adecuado para comprender la base del conocimiento turístico.

\section{Transdisciplinariedad del Turismo}

La transdisciplinariedad implica la generación de un conocimiento nuevo que surge de una serie de disciplinas y objetos de estudio que se concentran, sin ninguna jerarquización específica, lo que algunos autores denominan epistemología (Urdaneta, 2009), por ello es imperante la construcción de métodos capaces de explicar la complejidad que caracteriza al turismo, sin embargo, cabe destacar que este escenario aún no ha sido alcanzado debido a lo difícil que ha sido superar el abordaje multidisciplinario que lo ha caracterizado. Es evidente que el abordaje transdisciplinario lograría entender la esencia del conocimiento turístico. 
El turismo es un fenómeno integral, complejo y multidimensional, que resulta de la interrelación de múltiples actores, contextos (espaciales y temporales) y niveles de actividad, por lo que es necesario considerar las preguntas y problemáticas de manera transversal y dinámica. Entonces es importante plantear formas que conlleven a la reflexión e integración de las mismas (Campodónico y Chalar, 2017).

Es imperante comprender el conjunto de disciplina que están involucradas con el turismo y lo que cada una de ellas aportan al conocimiento de este fenómeno tan versátil, complejo y dinámico, el cual requiere de una crítica reflexiva que permita entender su esencia, por eso el abordaje disciplinario a considerar en su estudio debe ir más allá de la multi e interdisciplinariedad.

La mayoría de los estudios que se han realizado en turismo parten de disciplinas ya fortalecidas (economía, sociología, geografía, antropología, entre otras), por lo que es relevante destacar que a la hora de ofrecer una explicación científica todas esas disciplinas y otras se entrelazan, siendo esto uno de los motivos por los que hay un atraso en la realización de marcos conceptuales, ya que las perspectivas disciplinares no permiten alcanzar una visión integral. Por lo tanto, el turismo es un área de conocimiento transversal, que no cuenta con una malla disciplinar establecida siendo esto la complejidad que el investigador en turismo debe afrontar (Campodónico y Chalar, 2010).

Por ende, los estudiosos deben tomar en cuenta como se enlazan las disciplinas que abarcan el turismo, y la complejidad y transversalidad que lo caracteriza a fin de lograr una visión integral en la interpretación del turismo, lo cual generará un marco conceptual propio del turismo, y una malla que trascienda las disciplinas. Un ejemplo de la transversalidad dentro del turismo, se puede plantear considerando un determinado objetivo: Promover turísticamente un atractivo natural, para lograrlo entran en juego diversas disciplinas como son la ecología (es necesario tomar en cuenta la preservación y conservación del atractivo), la geografía (es necesario conocer las características climáticas, el área geográfica que lo comprenden), la matemática y estadística (se debe prever la capacidad de carga del atractivo), la política (acciones a desarrollar para promover el mismo), entre otras. No podemos considerar promover el atractivo sin tomar en cuenta cada una de las disciplinas, entrelazarlas e interrelacionarlas, puesto que, si se cumple el objetivo considerando una sola disciplina o la interrelación de dos de ellas, la realidad estaría sesgada, se obviarían diversos elementos, por lo tanto, se debe considerar de forma conjunta, transversal para así poder tener una visión integral de la realidad turística.

Es necesario superar la interdisciplinariedad y la multidisciplinariedad del turismo. La visión transdisciplinaria comprende una forma distinta de percibir, ver y pensar la realidad puesto que parte de un paradigma complejo donde está contenido sus tres principios que son: Dialógico, Recursividad Organizacional y Hologramático (Morín, 2001), los cuales son evidentes en el turismo.

En cuanto al principio dialógico, se fundamenta en la agrupación compleja (complementaria, antagonista) de eventos necesarios juntos para el funcionamiento y desarrollo de un fenómeno establecido, en el caso del turismo este principio se ve reflejado a través de los procesos antagónicos tales como: impactos negativos en el medio ambiente / beneficios económicos; aculturación / rescate del patrimonio histórico. Por su parte, la recursividad organizacional se refiere a que son las interacciones e interrelaciones entre los sujetos las que crean la sociedad, pero es la sociedad la que crea al sujeto; en el turismo, este principio se 
evidencia en los turistas y la diversidad de atractivos, servicios, equipamientos, instalaciones turísticas, donde el turista requiere de éstos para satisfacer sus deseos, pero a su vez esos atractivos, servicios, equipamientos e instalaciones se transforman y adaptan para la satisfacción del turista (La Rosa, 2012).

Por último, el hologramático refleja que no solo la parte está en el todo, sino que el todo está en la parte, cada elemento que conforma el sistema turístico abarca la totalidad de su información, por lo tanto, cada parte debe trabajar coordinadamente puesto que, el comportamiento de cada elemento incidirá en el resto de los elementos por lo que la coordinación es relevante dentro de cualquier sistema, en especial dentro del sistema turístico.

Esta complejidad que caracteriza al turismo, debe ser abordada desde la transdisciplinariedad, ya que el conjunto de procesos dinámicos que involucra el turismo, tiene que ser comprendido desde las distintas visiones y niveles que abarcan la realidad. Por ende, dentro de la transdisciplina la investigación que se desarrollaría será complementaria a la disciplinar (que se relaciona con un solo nivel de la realidad) pero muy diferente a ella, ya que en la investigación transdisciplinaria es evidente una dinámica originada por la acción simultanea de diversos niveles de la realidad (Campodónico y Chalar, 2010).

Lo transdisciplinario pretende ir más allá de multi e interdisciplinario, busca vencer la fragmentación, división y parcelación del conocimiento que revelan las disciplinas particulares. Las investigaciones transdisciplinarias hacen en la convergencia de saberes, en su interacción, interrelación e integración mutua o en su alteración o progreso (Campodónico y Chalar, 2010), por lo tanto, ésta representa la forma más adecuada para abordar el conocimiento turístico.

La transdisciplinariedad implica que un objeto de estudio puede ser abordado por diversas disciplinas que se interrelacionan e interceptan unas a otras con sus discursos, saberes, explicaciones e interpretaciones (Campodónico y Chalar, 2010). Esto es evidente en el turismo, donde varias disciplinas convergen entre sí, por tanto, ellas con sus conocimientos, disertaciones, debates y explicaciones permitirán tener una visión integral del turismo, por ende, comprender la esencia del conocimiento turístico.

Por ejemplo, en un determinado destino se pretende planificar el desarrollo del turismo, para lograrlo es indispensable abordarlo de manera integral: se debe conocer las motivaciones del turista (sociología), determinar los principales atractivos naturales y culturales (ecología, geografía, antropología), así como los posibles impactos que generaría los encuentros entre la comunidad receptora y los turistas (geografía, sociología, antropología, psicología, ecología); también, se deben estimar los ingresos y gastos que se pueden originar (estadística, economía) y plantear las acciones gubernamentales que se pueden ejecutar (política). Como se puede apreciar, diversas disciplinas hacen vida en el turismo por lo que es necesaria la transversalidad de las mismas, para poder comprenderlo, es imperante la trasndisciplinarieadad. Este enfoque puede ser abordado en el estudio del turismo para así comprender su esencia, el turismo es un fenómeno transdisciplinario por la multiplicidad de áreas de conocimiento que en él convergen. El ámbito dinámico en el cual está inmerso el turismo precisa de un pensamiento complejo, crítico y reflexivo, que permita generar un cuerpo teórico, marco conceptual y la trascendencia del sujeto al objeto de estudio (Panosso, 2007), sobre esto se genera el enfoque transdisciplinar, el cual representa el abordaje disciplinario más adecuado para el estudio del turismo, a través de éste se podrá comprender mejor su esencia (ver figura 3). 
Figura 3. Transdisciplinariedad del Turismo.

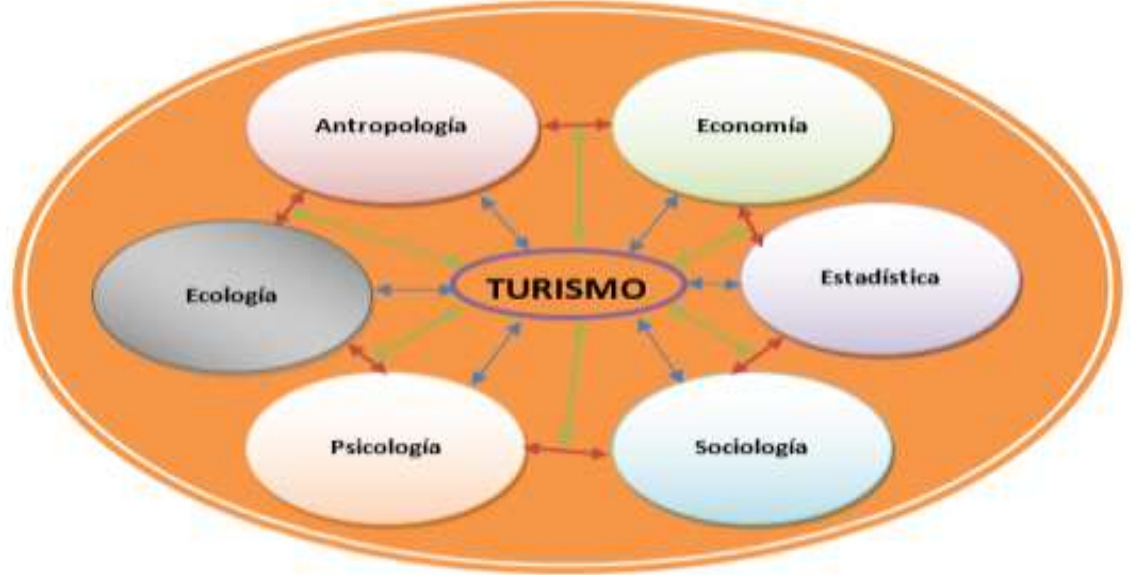

Fuente: Elaboración Propia.

\section{Conclusiones}

Teóricamente, no existe uniformidad a la hora de definir el turismo debido a las diversas implicaciones que lo caracterizan y las distintas consideraciones a las que puede ser sometido en dependencia de la disciplina que lo analice.

En la actualidad, el turismo ha sido analizado desde diversos ámbitos, sin embargo, es común que la mayoría de los estudios se enfocan en áreas específicas, lo que no permite comprender su naturaleza pues solo se visualiza la parcialidad, particularidad y especificidad de la disciplina de estudio que lo aborda, sin mostrar lo integral y complejo del turismo, es decir, los estudios se han centrado en la multidisciplinariedad del turismo, sin manifestar la relación existente entre disciplinas.

Desde la economía, sociología, psicología, estadísticas, entre otras disciplinas, se han realizado innumerables estudios sobre el turismo, que solo muestran un aspecto particular de su realidad, enfocándose en las estrategias y metodologías propias de la disciplina, mostrando resultados sesgados sobre la base del conocimiento turístico.

En cuanto a la interdisciplinariedad del turismo, son pocos los estudios que se han realizado siendo este uno de los posibles abordajes adecuado para su estudio, donde las teorías y metodologías de cada disciplina pueden ayudar a comprenderlo, así puedan surgir las bases conceptuales, teóricas y metodológicas propias del turismo, de una forma integral. A pesar de lo beneficioso que es la interdisciplinariedad, está surgiendo una nueva forma para abordar el estudio del turismo, el cual permite analizarlo desde la totalidad, complejidad y complementariedad que lo caracteriza, eso es la transdisciplinariedad.

El abordaje transdisciplinario destaca la transversalidad que debe existir entre las distintas disciplinas que se relacionan y abordan el estudio del turismo, enfocándose en la dinámica que se origina por la acción simultánea de los diversos niveles de realidad que se evidencian en el turismo. Por ende, se considera que este es el abordaje disciplinario acorde a 
utilizar para el estudio del turismo, ya que éste permitirá comprender su naturaleza, de una forma integral, complementaria y holística.

\section{Referencias Bibliográficas}

Acosta, Jorge y García, Evelyn (2016). Análisis epistemológico de la investigación turística.

Revista Ciencia y Tecnología. Volumen 2, №12, Ecuador (Pp. 43-52).

Ascanio, Alfredo (2010). El objeto del turismo. ¿Una posible ciencia social de los viajes?. Pasos.

Revista de Turismo y Patrimonio Cultural. Volumen 8, № 4, España (Pp. 633-641). DOI: https://doi.org/10.25145/j.pasos.2010.08.054

Campodónico, Rossana y Chalar, Luis (2010). Turismo: Una ciencia en construcción. Memoria del Anais do VI Seminario de pesquisa en turismo do Mercosol. Rio Grande del Sur (Universidad de Caixas do Sul). Brasil.

Campodónico, Rossana y Chalar, Luis. (2017). El abordaje interdisciplinario del turismo. El campo de análisis TEMA como propuesta metodológica. Estudios y perspectivas en turismo. Volumen 26, No2. Argentina (Pp. 461- 477). Recuperado de: https://www.estudiosenturismo.com.ar/PDF/V26/N02/v26n2a12\%20.pdf

Castaño, José. (2005). Psicología social de los viajes y del turismo. Editorial Thomson. España.

Castillo, Marcelino. (2005). Inter, multidisciplina y/o hibridación en los estudios socioculturales del turismo. ?. Pasos. Revista de Turismo y Patrimonio Cultural. Volumen 3, №2. España (Pp. 229 - 243). DOI: https://doi.org/10.25145/j.pasos.2005.03.018

Castillo, Marcelino y Lozano, Maribel (2006). Apuntes para la investigación turística. Editorial Cuerpo académico de turismo. Universidad de Quintana Roo. México.

Dos Santos, Joao (2009). Questões teóricas expressam riqueza e pobreza no debate epistemológico do fenômeno turístico: uma ciência em construção". Revista Espaço Acadêmico. Año 8, No96. Brasil (Pp. 1 - 7). Recuperado de: http://www.periodicos.uem.br/ojs/index.php/EspacoAcademico/article/view/42297

Goeldner, Charles; Ritchie, JR Brent; Mclntosch, Robert. (2002). Turismo: principios, prácticas e filosofías. Editorial Bookman. Brasil.

Hessen, Johannes (2001). Teoría del Conocimiento. Editorial Panamericana. Colombia.

La Rosa, Yvón (2012). Aproximación a una reinterpretación teórica del turismo desde otras lógicas del pensamiento. Magister Scientiarum en Turismo. Universidad de Oriente, Núcleo Nueva Esparta, Venezuela.

Martínez, José (2013). El turismo como ciencia. Sexto Congreso Internacional sobre Historias y Ciencias Sociales. Universidad de Málaga. España.

Martini, Marutschka (2013). El origen del conocimiento: El lugar de la experiencia y de la razón en la génesis del conocimiento del turismo. Estudios y perspectivas en Turismo. Volumen 22, N05. Argentina (Pp. 985-1001). Recuperado de: http://www.estudiosenturismo.com.ar/PDF/V22/N05/v22n5a10.doc.pdf

Morales, Samuel (2011). ¿Investigación interdisciplinaria y "Sostenible"?. Una lectura sobre el estadio de la cuestión en la investigación en el turismo en América Latina y algunas reflexiones para su mejora. Ponencia Congreso de Investigación en Mar de Plata. Facultad Latinoamericana en Ciencias Sociales. Argentina.

Moreira, Paulo; Lopes, Ligia y De Abreu, Maurilio (2015). El turismo como núcleo de estudio interdisciplinario: $[R e]$ construcción de los procedimientos y adecuaciones metodológicas. 
Estudios y perspectivas en Turismo. Volumen 24, №3. Argentina (Pp. 450-469). Recuperado de: https://www.estudiosenturismo.com.ar/PDF/V24/N03/v24n3a01.pdf

Morín, Edgar (2001). La cabeza bien puesta. Repensar la reforma reformar el pensamiento. Ediciones Nueva Visión. Argentina.

Panosso, Alexander (2007). Filosofía del turismo una propuesta epistemológica. Estudios y perspectivas en turismo. Volumen 16, №4. Argentina (Pp. 389-402). Recuperado de: https://www.estudiosenturismo.com.ar/search/PDF/v16n4a01.pdf

Rocha Centeno, Rogelio (1992). Metodología de la investigación aplicada al turismo. Casos Prácticos. Editorial Trillas. México.

Urdaneta, Carmen (2009). Reflexiones sobre Epistemología del Turismo, en Castillo N., Marcelino y Alexandre Panosso Netto. Epistemología del Turismo. Estudios Críticos, p.5361. Editorial Trillas. México. 